Tohoku J. exp. Med., 1987, 151, 89-95

\title{
Quantitative NBT Reduction Test with or without Stimulation of Peripheral Polymorphonuclear Cells from Pregnant Women
}

\author{
Kunihiro Okamura, Yukinori Yonemoto, Hisashi \\ Higashimwai and Akira Yajima \\ Department of Obstetrics and Gynecology, Tohoku \\ University School of Medicine, Sendai 980
}

\begin{abstract}
Okamura, K., Yonemoto, Y., Higashirwai, H. and Yajima, A. Quantitative NBT Reduction Test with or without Stimulation of Peripheral Polymorphonuclear Cells from Pregnant Women. Tohoku J. exp. Med., 1987, 151 (1), 89-95-We performed the nitroblue tetrazolium (NBT) reduction test to investigate the function of polymorphonuclear cells (PMN) from prenatal, intrapartal, postpartal and nonpregnant women with or without stimulation by phorbol myristate acetate (PMN). The capacity for NBT reduction of PMN was significantly increased in pregnancy, followed by a further increases during labor without any stimulation. On the other hand, when stimulated with PMA, there were no differences between pregnancy and labor in the reactivities of PMNs, although they were still significantly higher than those of nonpregnant women. Kallikrein increased the activity of PMN in an in vitro study, suggesting that it may be associated with the change in PMN function in labor. - pregnancy; leucocyte; NBT reduction test
\end{abstract}

It has been reported that maternal immunity, particularly cell-mediated immunity in depressed during pregnancy (Purtilo et al. 1972 ; Covelli and Wilson 1987), probably in order to adapt the mother to acceptance of the allograft i.e., the fetus. In spite of the depression of cellular immunity mother is not especially vulnerable to pathogenic organisms from the clinical point of view. This clinical observation may imply the presence of some compensating mechanisms in the maternal immunosurveillance system. Leucocytosis during pregnancy may be one such compensating mechanism, although the significance of these changes are not fully understood. There have been some reports that leucocyte activity is increased during pregnancy (Mitchell et al. 1970; Selvaraj et al. 1982). However, the matter is still controversial (E1-Maallem and Fletcher 1980). Enhancement of NBT reduction by normal cells in vitro has been clearly shown to be associated with the phagocytic process. Numerous publications have appeared regarding the usefulness of this test. In order to examine the function of the leucocyte, we

Received September 4, 1986 ; accepted for publication December 2, 1986. 
performed the nitro blue tetrazolium (NBT) test with or without stimulation of leucocytes obtained from the peripheral blood of perinatal, intrapartal and postpartum women.

\section{Materials and Methods}

Heparinized peripheral blood was obtained from healthy nonpregnant, perinatal, intrapartum (in the second stage of labor and puerperal (at the 6th postpartum day) women. Buffy-coat first obtained by the gelatin sedimentation method was washed twice with phosphate buffered saline (PBS), overlayered on Ficoll-Conray solution $(\mathrm{d}=1.077)$ and centrifuged at $400 \mathrm{~g}$ for $30 \mathrm{~min}$. The pellet which was over $95 \%$ enriched in PMN cells, was washed three times again with PBS and adjusted to $2 \times 10^{6} / \mathrm{ml}$ in PBS. According to our preliminary examination NBT reduced activity of the leucocytes was maintained at $4{ }^{\circ} \mathrm{C}$ for at least $4 \mathrm{hr}$.

NBT reduction test; The activity of NBT reduction was quantitatively measured according to the method of Imaizumi and Breitman (Imaizumi and Breitman 1986). A million of leucocytes in $0.5 \mathrm{ml}$ PBS were added to $0.5 \mathrm{ml}$ of $0.1 \%$ NBT solution and incubated while being gently shaken at $37^{\circ} \mathrm{C}$ for $25 \mathrm{~min}$. After halting the reaction by immersion in an ice cold bath, formazan was recovered as a pellet by centrifugation at 1,000 $\mathrm{g}$ for $10 \mathrm{~min}$. The formazan was washed twice with $0.1 \mathrm{~N} \mathrm{HCl}$. Three milliliters of $N$, $N$-dimethylformamide and $3 \mu \mathrm{l}$ of $1 \mathrm{~N} \mathrm{NaOH}$ were used to extract the formazan from the pellets. The extract was immediately read the optical density at a wavelength of $710 \mathrm{~nm}$. The formazan yield was calculated according to our observation that $1 \mathrm{nmole} / \mathrm{ml}$ formazan should correspond to an optical density $0.054 \pm 0.003$. Phorbol myristate acetate, PMA (Sigma, St. Louis, MO, USA), which is a strong activator of NBT reduction, was diluted to the concentration of $10 \mu \mathrm{l} / \mathrm{ml}$ with dimethyl sulfoxide and stored until used. Oxytocin was obtained from the Sankyo Pharmaceutical Co. Ltd. (Tokyo), prostaglandin $\mathrm{F}_{2} \alpha$ from Ono Pharmaceutical Co. Ltd. (Osaka) and kallikrein was purchased from Bayer Co. Ltd. (Osaka). These chemicals were added in several concentrations dissolved in PBS at the start of NBT test.

\section{Results}

The formazan yield from $10^{6}$ polymophonuclear (PMN) cells without any stimulation was $5.0 \pm 0.6$ nmole (mean \pm s.D.) in nonpregnant women. In pregnant women, the yield was $6.1 \pm 0.6$ nmole (mean \pm s.D.). In pregnant women, the yield was $6.1 \pm 1.4$ nmole in the first half of pregnancy and $5.9 \pm 0.6$ in the second half, both of which were significantly high $(p<0.01)$ compared with the value in nonpregnant women. In labor there was a much greater increase in NBT activity than in the prenatal period $(p<0.01)$. The formazan yield returned to the prenatal level by the 6th postpartum day, although it was still significantly higher than that in nonpregnant women (Fig. 1). When the activity of NBT reduction in PMN cells was stimulated by PMA, there were no differences in the formazan yield between the first half of pregnancy $\left(28.0 \pm 6.1 \mathrm{nmole} / 10^{6}\right.$ cells, mean \pm s.D. $)$, the second half of pregnancy $(30.5 \pm 4.2)$, the second stage of labor $(30.3 \pm 4.9)$ and the 6th postpartum day. However, each of these was higher than that in nonpregnant women $(22.0 \pm 6.7)$ (Fig. 2).

Since NBT reduction activity in pregnant women was remarkably enhanced 


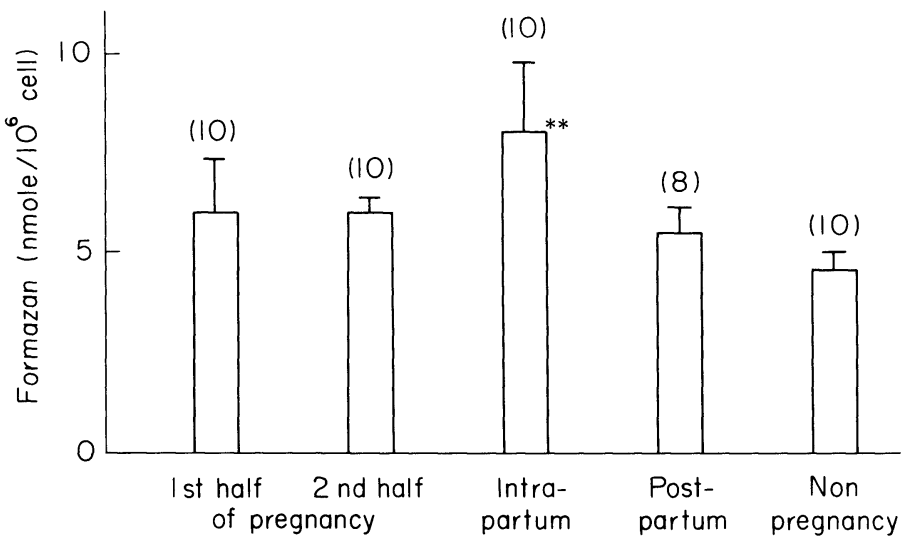

Fig. 1. Formazan yield in PMNs from pregnant and nonpregnant women without stimulation. Mean values and s.D. are shown. Number of subjects are given in parentheses. ${ }^{* *} p<0.01$ against the value in non-pregnant women.

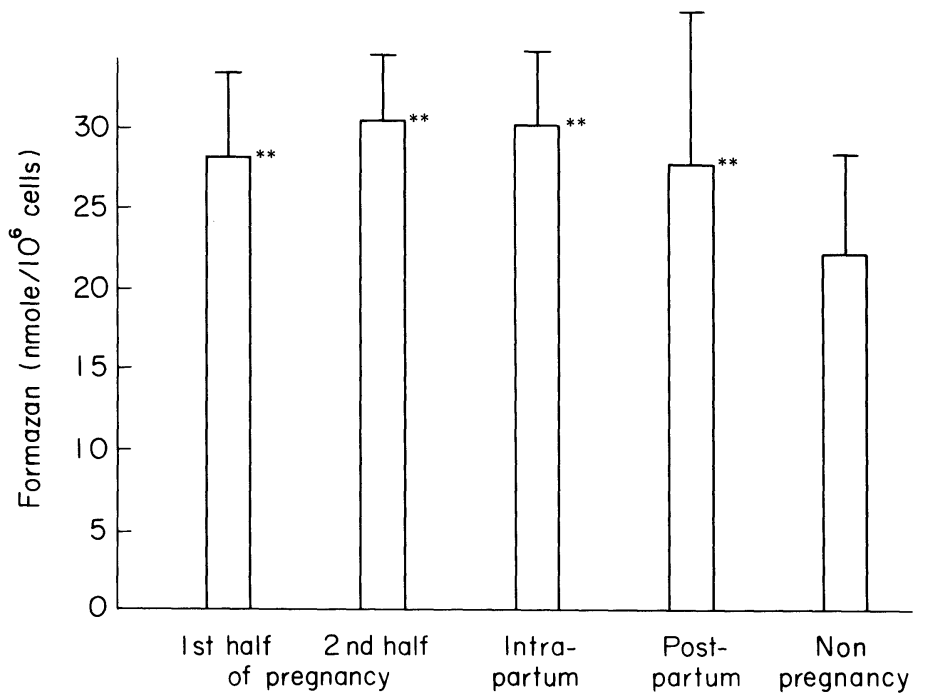

Fig. 2. Formazan yield in PMNs from pregnant and nonpregnant women with stimulation by PMA. Mean values and S.D. of 10 subjects are shown. PMA : $0.1 \mu \mathrm{g} / 10^{6}$ cells. ${ }^{* *} p<0.01$ against the value in non-pregnant women.

during labor, we tested for factors, specially humoral, which could be involved in the change in PMN cell activity. In this study we chose oxytocin, prostaglandin $\mathrm{F}_{2} \alpha$ and kallikrein as possible factors. Neither oxytocin nor prostaglandin $\mathrm{F}_{2} \alpha$ enhanced NBT reduction in physiological dosages, whereas kallikrein enhanced it with dosages over 0.1 units (Figs. 3, 4, 5 and 6). 


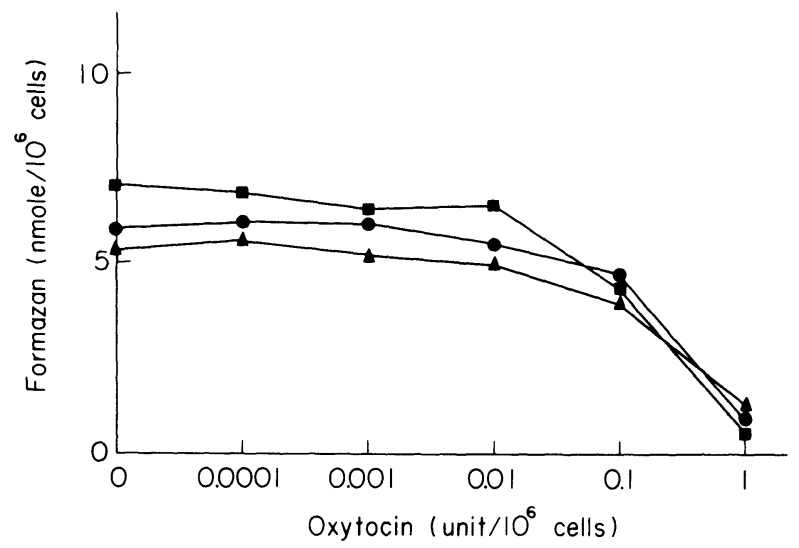

Fig. 3. Relation between formazan yield in PMNs and oxytocin concentration. Values of 3 subjects are presented.

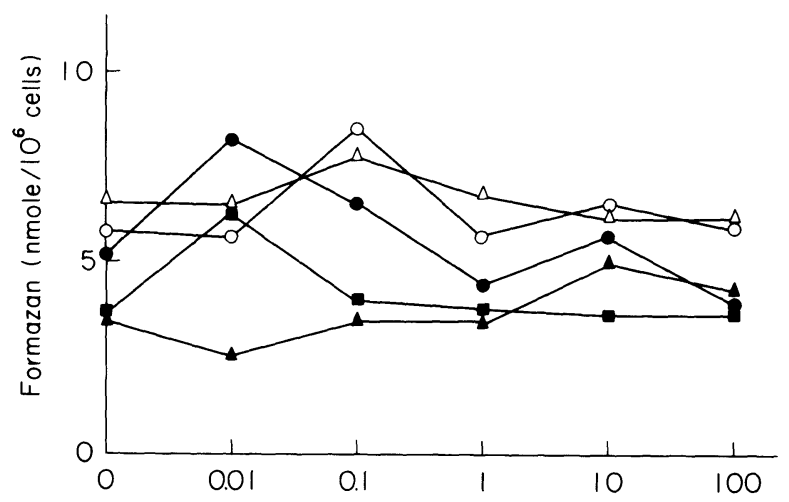

Fig. 4. Relation between formazan yield in PMNs and prostaglandin $\mathrm{F}_{2} \alpha$ concentration. Values of 5 subjects are presented.

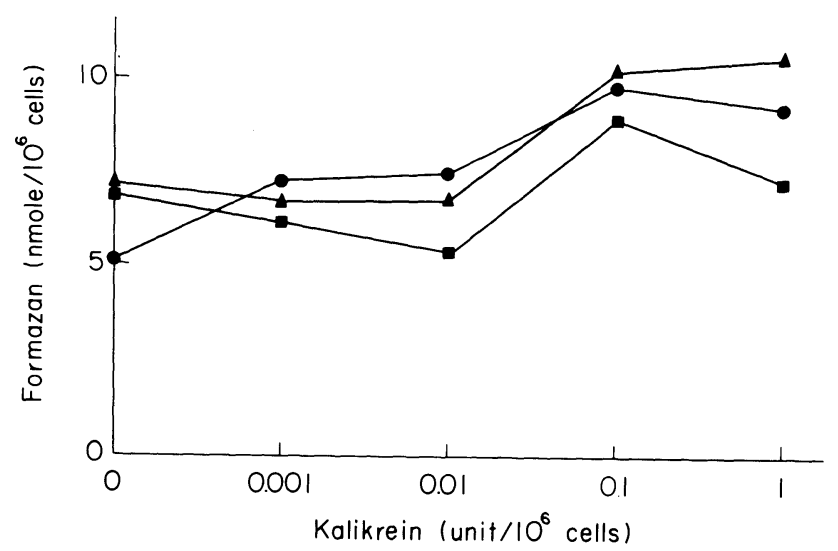

Fig. 5. Relation between formazan yield in PMNs and kallikrein concentrations. Values of 3 subjects are presented. 


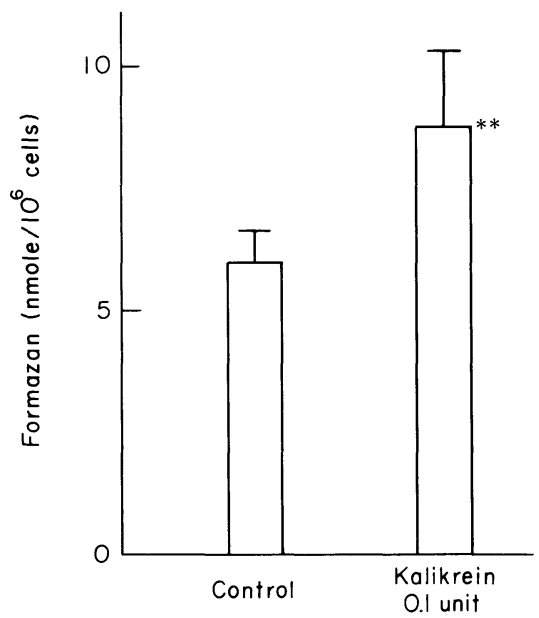

Fig. 6. Increases of formazan yield in PMNs by kallikrein. Mean values \pm S.D. of 10 subjects are shown. ${ }^{* *} p<0.01$ against control.

Discussion

It is known that an increases in the number of circulating PMN cells, which play an important part in the defense mechanism, takes place in pregnant women (Fleming 1975). In addition, there have been some reports showing that function as well as cell number are altered during pregnancy. However, it remains controversial whether or not the function of PMN in enhanced. Mitchell et al. (1970) reported in a series of their contribution to leucocyte function in pregnancy that the phagocytic and bactericidal activity of leucocytes from pregnant women was enhanced. It would seem to be useful from clinical point of view to know to what extent pregnant women are able to react to contact with bacterial organisms. On the other hand, a contrary finding was reported by El-Maallem and Fletcher (1980). In the present paper, we showed that the activity, as tested by NBT reduction of leucocytes during pregnancy is slightly enhanced, without prior stimulation, from an early period of pregnancy, which accords with the observations of Mitchell et al. (1970). Estrogen and corticosteroid have been reported as being factors possibly involved in this enhancement (Jacobs et al. 1973 ; Goldstein et al. 1976). These substances which are increased in blood as pregnancy progresses, may continue stimulating leucocyte activity throughout the period of pregnancy. According to the present results, however, there remained a difference between pregnant and nonpregnant women, even when stimulated to a rather high degree with PMA. Although the biological significance of the fact that NBT reduction of leucocytes in pregnant women is enhanced is not well understood, this fact may imply that leucocytes in pregnant women are altered in some intrinsic way resulting in a capacity to more vigrorously fight microorganisms than are those of nonpregnant women. This may be regarded as one piece of evidence 
suggesting the existence of an elaborate defensive system in pregnant women, in spite of the depression of cellular immunity.

In labor, on the other hand, NBT reduction in peripheral leucocytes was further enhanced beyond that in the prenatal period, even without strong stimulation. There have been many reports that enzymes in peripheral leucocytes, such as alkalinephosphatase and peroxidase, are increased in the prenatal period and reach a peak in labor.

Our study showed that when the leucocytes were intensively stimulated with PMA, not only in the prenatal period but also in labor, the NBT reduction activity in each period did not differ. The total capacity of leucocytes for NBT reduction, when fully activated, is almost constant during pregnancy. In the light of these results, we speculate that leucocytes which have already attained increased reactivity in the prenatal period as mentioned earlier, than in the nonpregnant state may gain no further intrinsic change further increasing NBT reduction, but may simply react to some stimuli possibly generated in labor. Accordingly, the preserved capacity of leucocytes in pregnancy may be spent in labor. We performed in vitro studies of oxytocin, prostaglandin $\mathrm{F}_{2} \alpha$ and kallikrein as possible substances involved in the enhancement of PMN function. Since in our study only kallikrein produced a stimulating effect in the reduction test, we think that it may be associated with the dynamic change of leucocyte activity in labor. However, the biological significance of the fact that leucocyte activity increases in labor is difficult to explain from our results alone.

\section{References}

1) Covelli, H.D. \& Wilson, R.T. (1987) Immunologic and medical considerations in tuberculine sensitized pregnant patients. Amer. J. Obstet. Gynec., 132, 256.

2) El-Maallem, H. \& Fletcher, J. (1980) Impaired neutrophil function and myeloperoxidase deficiency in pregnancy. Brit. J. Haemat., 44, 375-381.

3) Fleming, A.F. (1975) Haematological changes in pregnancy. Clin. Obstet. Gynec., 2, 269-283.

4) Goldstein, I.M., Roos, D., Weissmann, G. \& Kaplan, H.B. (1976) Influence of corticosteroids on human polymorphonuclear leukocyte function in vitro. Inflammation, $\mathbf{1}$, 305-315.

5) Imaizumi, M. \& Breitman, T.R. (1986) A combination of a T cell-derived lymphokine differentiation-inducing activity and a physiologic concentration of retinoic acid induces HL-60 to differentiate to cells with functional chemotactic peptide receptors. Blood, 67, 1273-1280.

6) Jacobs, A.A., Selvaraj, R.J., Strauss, R.R., Paul, B.B., Mitchell, G.W., Jr. \& Sbarra, A.J. (1973) The role of the phagocyte in host-parasite interaction. XXXIX. Stimulation of bactericidal activity of myeloperoxidasecontaining leukocytic fractions by estrogens. Amer. J. Obstet. Gynec., 117, 671-678.

7) Mitchell, G.W., Jr., Jacobs, A.A., Haddad, V., Paul, B.B., Strauss, R.R. \& Sbarra, A. J. (1970) The role of the phagocyte in host-parasite interaction. XXV. Metabolic and bactericidal activities of leukocytes from pregnant women. Amer. J. Obstet. Gynec., 108, 805-813.

8) Purtilo, D.T.H., Hallgren, H.M. \& Ynnis, E.J. (1972) Depressed maternal lymphocyte 
response to phytohaemagglutinin in human pregnancy. Lancet, 1, 769.

9) Selvaraj, R.J., Sbarra, A.J., Thomas, G.B., Cetrulo, C.L. \& Mitchell, G.W., Jr. (1982) A microtechnique for studying chemiluminescence response of phagocytes using whole blood and its application to the evaluation of phagocyte in pregnancy. $J$. reticuloendoth. Soc., 31, 3-16. 